\title{
Usefulness of sheathless guiding catheters in patients with upper extremity vascular anomalies
}

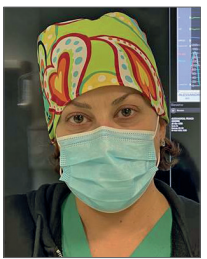

Cristina Aurigemma ${ }^{1}, \mathrm{MD}, \mathrm{PhD}$; Osama Shoeib ${ }^{2}, \mathrm{MD}, \mathrm{PhD}$; Francesco Burzotta ${ }^{1,3, *} \mathrm{MD}, \mathrm{PhD}$;

Antonio Maria Leone ${ }^{1}, \mathrm{MD}, \mathrm{PhD}$; Giampaolo Niccoli ${ }^{1,3}, \mathrm{MD}, \mathrm{PhD}$; Italo Porto ${ }^{1}, \mathrm{MD}, \mathrm{PhD}$;

Giulio Russo ${ }^{1}$; Diana Verdirosi ${ }^{1}$; Carlo Trani ${ }^{1,3}, \mathrm{MD}$

1. Institute of Cardiology, Fondazione Policlinico Universitario A. Gemelli IRCSS, Rome, Italy; 2. Cardiology Department, Tanta University, Tanta, Egypt; 3. Institute of Cardiology Università Cattolica del Sacro Cuore, Rome, Italy

C. Aurigemma and S. Osama have equally contributed to this manuscript.

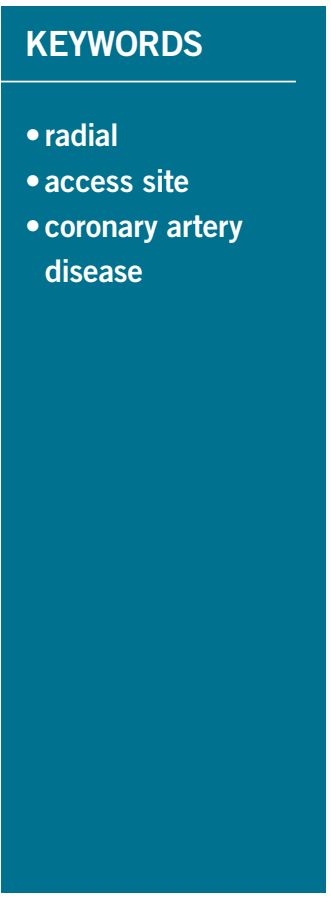

\section{Abstract}

Aims: The transradial approach (TRA) reduces hospitalisation and access-site complications as compared to the transfemoral approach. Nevertheless, the TRA technical failure rate is significantly higher compared to the transfemoral approach. The high failure rate of TRA is due to a series of factors. In particular, a wide range of anatomic vascular variants hindering procedural success may be present in patients undergoing TRA procedures.

Methods and results: In our retrospective observational study, 1,596 consecutive patients with upper limb vascular anomalies underwent TRA between January 2006 and July 2017. We evaluate the usefulness of the sheathless guiding catheter system (SG) as compared to the conventional guiding catheter (CG). The primary study endpoint was the "procedural success" defined as successful transradial procedure (both selective cannulation of the coronary ostium in the diagnostic procedure and successful stent delivery in the interventional procedure) without access change. All SG procedures were successful, whereas only 1,274 (86\%) CG procedures were successfully performed $(\mathrm{p}=0.0001)$. At multivariable analysis, age $(\mathrm{p}=0.001)$ and sheathless catheter use $(\mathrm{p}=0.001)$ were independent predictors of procedural success.

Conclusions: The sheathless GC is a safe and useful system not only for small radial access but also in the presence of upper vascular anomalies and it can be used in PCI and diagnostic procedures.

*Corresponding author: Institute of Cardiology, Catholic University of the Sacred Heart, L.go A. Gemelli 1, 00168 Rome, Italy. E-mail: francesco.burzotta@unicatt.it 


\section{Abbrevations}

AV arteriovenous

GC guiding catheter

PCI percutaneous coronary intervention

TRA transradial approach

TFA transfemoral approach

\section{Introduction}

The transradial approach (TRA) for coronary diagnostic and interventional procedures reduces hospitalisation and access-site complications as compared to the transfemoral approach (TFA) $)^{1-2}$. Therefore, the TRA is now increasingly adopted not only for coronary diagnostic and interventional procedures but also for peripheral interventions ${ }^{3-8}$. Nevertheless, the TRA technical failure is significantly higher than that reported for the TFA ${ }^{2}$. Radial artery spasm and anatomic variants either of brachioradial and axillosubclavian anonymous arterial axis, or of the aortic arch, influence TRA technical procedural success. Device improvements have been developed to overcome these TRA limitations. Indeed a sheathless guiding catheter system (Eaucath, Asahi Intecc, Aichi, Japan), which does not require an introducer, has been developed to allow a gain in inner diameter, with a hydrophilic coating which covers the whole length of the sheathless device and reduces frictional forces, radial artery spasm and hindering in anatomic variants navigation (Figure 1) ${ }^{9}$.

In the present study we retrospectively evaluate the usefulness of the sheathless guiding catheter system in patients with upper arm vascular anomalies.

\section{Materials and methods STUDY DESIGN}

We conducted a retrospective observational study including consecutive patients with upper arm vascular anomalies who underwent

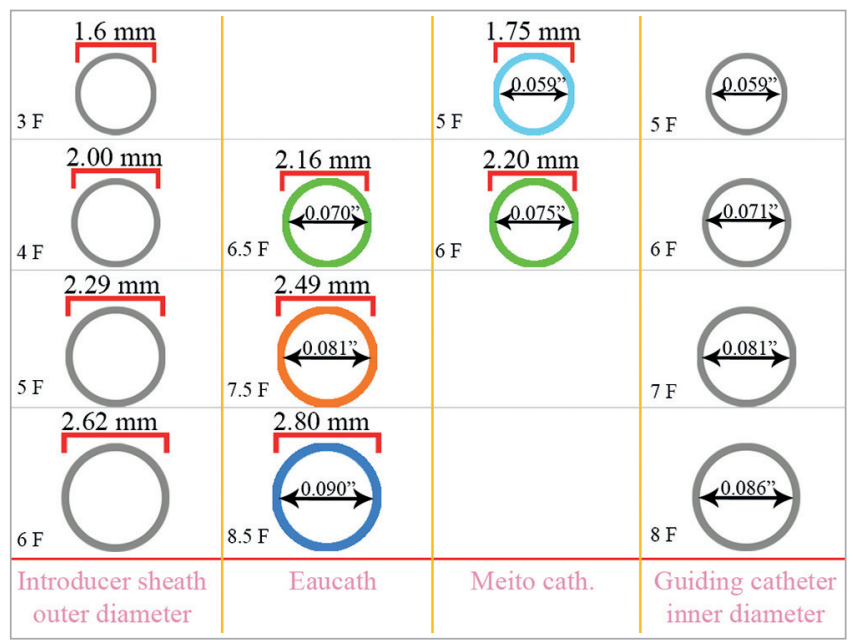

Figure 1. The sheathless guiding catheter system. The sheathless guiding catheter system (Asahi Intecc, Aichi, Japan), which does not require an introducer, was developed in order to allow a gain in inner diameter.
TRA procedures between January 2006 and July 2017 in a single tertiary high-volume centre (1,100 percutaneous coronary interventions per year). Clinical and procedural characteristics were prospectively collected for each patient and entered into a dedicated catheterisation laboratory database (Estensa, Esaote, Genoa, Italy) that had been previously proven to help assessing the role of EuroSCORE I and II in $\mathrm{PCI}^{10,11}$ and the safety of the TRA ${ }^{12}$.

\section{TRANSRADIAL APPROACH TECHNIQUE AND VASCULAR ANATOMIC VARIANT CLASSIFICATION}

The TRA was used in presence of a normal Allen test or, if abnormal, of a normal Barbeau test (based on oximetry and plethysmography $)^{13}$. Patients on dialysis, with previous coronary artery surgery using both internal mammary arteries, or in cardiogenic shock were excluded from the study. Catheterisation of the radial artery was performed with an arterial puncture kit (with plastic cannula and hydrophilic wire) and long $(25 \mathrm{~cm})$ hydrophilic sheath (Radifocus ${ }^{\circledR}$, Terumo Corp., Tokyo, Japan). A 5 or 6 French (Fr) sheath was used in diagnostic procedures, a 6 Fr sheath in coronary interventions. In diagnostic procedures, a 5,000 IU heparin bolus was administered through the sheath; in interventional procedures, a weight-adjusted heparin (100 IU/kg) bolus was administered and was followed by intravenous heparin boluses, if required, to maintain activated clotting time between 250 and 300 sec. Routine administration of vasodilator drugs was not performed and nitrates only were used in the case of radial artery spasm. Retrograde arterial angiography was performed (from the cannula, from the sheath or from the catheter) anytime difficulty was encountered during wire or catheter advancement/manipulation. Upper arm vascular anomalies were classified according to $\mathrm{ABC}$ operative classification ${ }^{14}$ (Figure 2):

- Group A: radial-brachial arterial axis

- Group B: axillary-subclavian-anonymous axis

- Group C: aortic arch

When an anatomic variant was recognised by angiography, some tricks, as previously provided ${ }^{15}$, or the sheathless guiding catheter (GC) system, were used to try to overcome the technical problems and complete the TRA procedure. The sheathless GC has a hydrophilic coating covering its whole length and a central dilator allowing smooth insertion into small and/or spastic radial arteries. The sheathless GC and central dilator were advanced over a 0.035 " guidewire to the ascending aorta. The central dilator and the 0.035 " guidewire were subsequently withdrawn a few centimetres from the ostium in order to allow safe intubation of the coronary artery. Because of its stiffness, extreme care had to be taken not to advance the central dilator close to the aortic valve. The decision to use a sheathless GC instead of a conventional catheter, and the selection of a specific shape, were left to the interventional cardiologist's discretion.

Clinical/angiographic data recordings, the need for crossover to other approaches and reason for access crossover, were prospectively recorded in a dedicated catheterisation laboratory database (Estensa, Esaote, Genoa, Italy). 


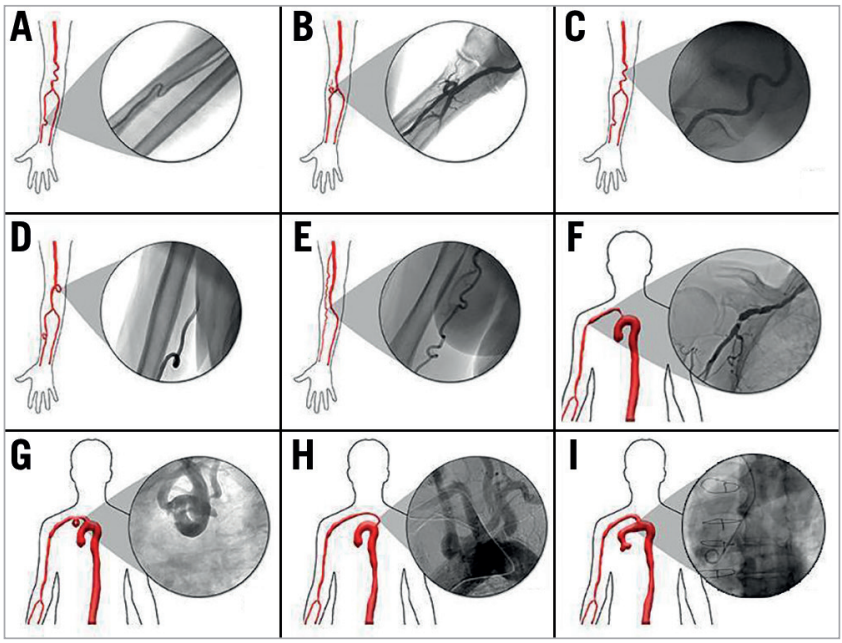

Figure 2. Upper limb vascular anomalies. Many upper limb vascular anomalies are associated with transradial approach failure and are classified according to ABC operative classification. Group A radial-brachial axis: radial tortuosity (Z shape) (A), radial loop $\left(360^{\circ}\right)(B)$, brachial tortuosity $(C)$, brachial loop (D), high radial origin (E). Group B axillary-subclavian-anonymous axis: high radial origin (E), axillary stenosis $(F)$, subclavian tortuosity $(G)$, subclavian lousoria (H). Group C: aortic arch: aortic elongation (I).

\section{STUDY ENDPOINTS}

The primary study endpoint was the "procedural success" defined as a successful transradial procedure (selective cannulation of both coronary ostium in the diagnostic procedure and successful stent delivery in the interventional procedure) without access change. Secondary endpoints were procedural time, amount of contrast, radiation amount, the use of additional techniques to complete the procedure (guiding catheter extension or buddy wire to increase the guiding catheter support) and incidence of vascular complications (pseudoaneurysm, AV fistula, dissection, minor and major haematoma, compartmental syndrome, perforation, occlusion).

\section{STATISTICAL ANALYSIS}

Continuous variables were reported as mean \pm standard deviation and compared with analysis of variance (Student's t test). Categorical variables were expressed as frequencies and compared with $\chi^{2}$ test. Normality of data was determined using the D'Agostino-Pearson test and verified using histogram plots. A two-sided p-value of 0.05 was considered significant in the Student's t test; a one-sided p-value of 0.05 was considered significant in $\chi^{2}$ test. Multivariable analysis to assess independent predictors of the primary procedural endpoint was performed using a backward elimination model which included the baseline clinical and anatomical variations as well as the type of catheter used to complete the procedure. Statistical analyses were conducted using SPSS v.18 (SPSS, Chicago, IL, USA).

\section{Results}

Out of 31,032 consecutive patients undergoing TRA at our centre during the study period, 1,596 (5\%) patients had upper arm vascular anomalies (Figure 3). The sheathless GC was systematically used in 112 patients (7\%), and in 2 patients it was used after failure of conventional diagnostic catheter. This constituted the SHEATHLESS group (SG), while the remaining 1,482 (93\%) patients, in whom conventional catheters were used, constituted the CONTROL group (CG). In 30 patients of the SG (27\%) and in 257 patients of the CG $(17 \%)$ only a diagnostic procedure was performed.

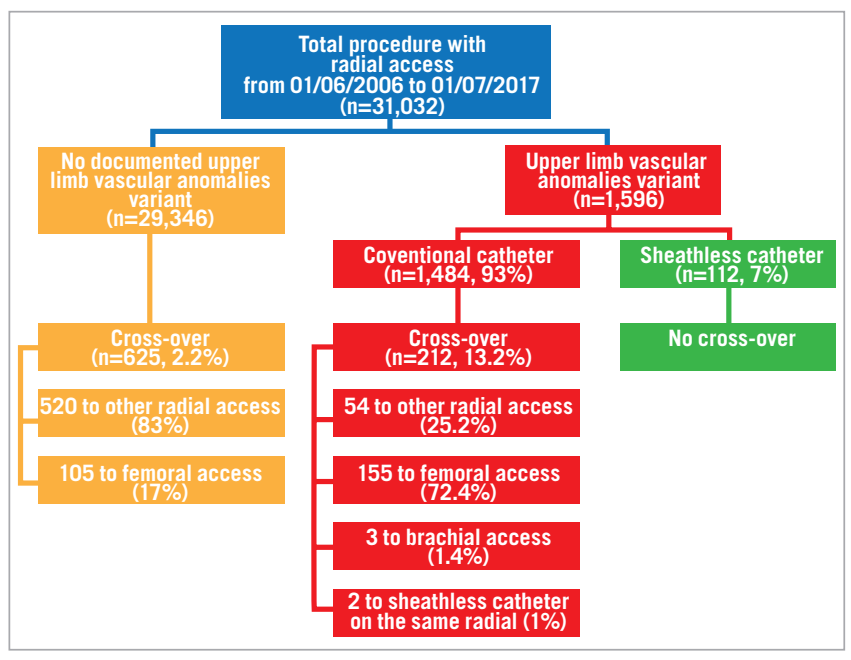

Figure 3. Study flow chart.

The baseline patient clinical characteristics are reported in Table 1, showing some differences in cardiovascular risk factors and cardiac clinical presentation between the two groups. In particular, female gender and hypertension was more common in the $\mathrm{SG}$ as compared to the CG ( $66 \%$ vs $47 \%$, p-value $=0.001$ and $81 \%$ vs $61 \%$, p-value $=0.001$, respectively). Moreover, acute coronary

Table 1. Baseline characteristics of the study group.

\begin{tabular}{|l|c|c|c|c|}
\hline Age & $\begin{array}{c}\text { All population } \\
(\mathbf{n = 1 , 5 9 6 )}\end{array}$ & $\begin{array}{c}\text { SG } \\
(\mathbf{n = 1 1 4})\end{array}$ & $\begin{array}{c}\text { CG } \\
(\mathbf{n = 1 , 4 8 2 )}\end{array}$ & p-value \\
\hline Sex (female) & $72 \pm 27$ & $74 \pm 11$ & $72 \pm 28$ & 0.1 \\
\hline DM & $772(48 \%)$ & $76(66 \%)$ & $696(47 \%)$ & $<0.001$ \\
\hline HTN & $275(17 \%)$ & $26(22 \%)$ & $249(17 \%)$ & 0.06 \\
\hline $\begin{array}{l}\text { History of peripheral } \\
\text { arterial disease }\end{array}$ & $979(61 \%)$ & $93(81 \%)$ & $886(61 \%)$ & $<0.001$ \\
\hline Clinical presentation & $35(2 \%)$ & $3(3 \%)$ & 32 & 0.4 \\
\hline \multicolumn{1}{|c|}{ STEMI } & $68(4 \%)$ & $16(14 \%)$ & $52(4 \%)$ & $<0.001$ \\
\hline \multicolumn{1}{|c|}{ NSTEMI } & $271(17 \%)$ & $30(26 \%)$ & $241(17 \%)$ & 0.006 \\
\hline \multicolumn{1}{|c|}{ CSA } & $594(37 \%)$ & $43(37 \%)$ & $551(38 \%)$ & 0.4 \\
\hline Previous PCI & $262(16 \%)$ & $23(20 \%)$ & $239(16 \%)$ & 0.1 \\
\hline Previous CABG & $139(9 \%)$ & $10(9 \%)$ & $129(9 \%)$ & 0.5 \\
\hline Diagnostic procedure & $1,230(77 \%)$ & $31(27 \%)$ & $1,199(81 \%)$ & 0.001 \\
\hline PCl & $339(21 \%)$ & $82(72 \%)$ & $257(17 \%)$ & 0.001 \\
\hline Peripheral intervention & $27(2 \%)$ & $1(1 \%)$ & $26(2 \%)$ & 0.7 \\
\hline CABG & & & & \\
\hline
\end{tabular}

CABG: coronary artery bypass graft; CSA: chronic stable angina; DM: diabetes mellitus; HTN: hypertension; NSTEMI: non-ST elevation myocardial infarction; PCI: percutaneous coronary intervention; STEMI: ST elevation myocardial infarction 
syndromes, either ST elevation myocardial infarction (STEMI) or non-ST elevation myocardial infarction (NSTEMI), were clinically present more frequently in the SG than in the CG (STEMI: $14 \%$ vs $4 \%$, p-value $=0.001$, NSTEMI: $26 \%$ vs $17 \%$, p-value $=0.006$ ).

The incidence of upper arm vascular anomalies in the two study groups is reported in Table 2. A high origin radial artery was a more frequent vascular anomaly in the $\mathrm{SG}$ as compared to the CG (46\% vs $31 \%$, p-value $=0.001)$, whereas in the CG, the incidence of radial tortuosity was more common as compared to the SG $(39 \%$ vs $27 \%$, p-value $=0.009)$. Percutaneous coronary interventions (PCI) were more frequent performed in the SG than in the $\mathrm{CG}(72 \%$ vs $17 \%$, p-value $=0.001)$ (Table 1$)$.

\section{PROCEDURAL OUTCOMES}

Procedural failure occurred in $212(13 \%)$ patients. Radial artery loop $360^{\circ}$ and brachial artery tortuosity were the most frequent anatomic variations in failed procedures $(p=0.001$ and $p=0.01$, respectively) (Table 2).

Procedural success was significantly different between the two study groups. In particular, all procedures were successful in the 112-patient SG (the 2 patients in which a sheathless GC was used after conventional catheter failure, were excluded from the procedural outcomes analysis), whereas only 1,274 procedures were successfully performed in the CG $(100 \%$ vs $86 \%$ CG; $p=0.0001)$. Procedural success was also significantly higher in the SG as compared to the CG in PCI procedures and in a PCI sub-analysis on conventional guiding catheter size.

At multivariable analysis, age $(\mathrm{p}=0.001)$ and sheathless catheter use $(p=0.001)$ were independent predictors of procedural success.

Procedure duration, fluoroscopic time and amount of contrast medium were significantly greater in the $\mathrm{SG}$ as compared to $\mathrm{CG}$ (Table 3). However, in a sub-analysis of PCI procedures, dose area product (DAP) and the amount of contrast medium were significantly lower in the SG as compared to the CG (Table 3). In PCI procedures, the sub-analysis according to conventional guiding catheter size has demonstrated a lower DAP and amount of contrast medium in the SG as compared to the 6 Fr CG, while only the procedural success was different between the SG and the 5 Fr CG (Table 4).

\section{Discussion}

The TRA is associated with a relevant reduction in hospitalisation and vascular complications ${ }^{1-2}$. However, the average technical

Table 2. Upper vascular anomalies in the two study groups and in successful and failed procedures.

\begin{tabular}{|c|c|c|c|c|c|c|}
\hline & $\begin{array}{c}S G \\
(n=114)\end{array}$ & $\begin{array}{c}\text { CG } \\
(n=1,482)\end{array}$ & $p$-value & $\begin{array}{c}\text { Procedural } \\
\text { success }(n=1,384)\end{array}$ & $\begin{array}{l}\text { Procedural failure } \\
\qquad(n=212)\end{array}$ & $p$-value \\
\hline A group & $97(85 \%)$ & $1,171(79 \%)$ & 0.1 & $1,098(97 \%)$ & $170(80 \%)$ & 0.8 \\
\hline Radial stenosis or atherosclerosis & $5(4 \%)$ & $60(4 \%)$ & 0.8 & $52(4 \%)$ & $13(6 \%)$ & 0.1 \\
\hline Radial occlusion & $2(2 \%)$ & $10(0.6 \%)$ & 0.2 & $8(0.5 \%)$ & $4(2 \%)$ & 0.06 \\
\hline Remnant radial artery & $53(46 \%)$ & $458(31 \%)$ & 0.001 & $445(32 \%)$ & $66(31 \%)$ & 0.8 \\
\hline Radial tortuosity $>45$ degree & $31(27 \%)$ & $584(39 \%)$ & 0.009 & $545(39 \%)$ & $70(33 \%)$ & 0.08 \\
\hline Radial loop $360^{\circ}$ & $15(12 \%)$ & $173(12 \%)$ & 0.6 & $129(9 \%)$ & $59(28 \%)$ & 0.0001 \\
\hline Brachial loop & $2(2 \%)$ & $34(2 \%)$ & 1 & $30(2 \%)$ & $6(3 \%)$ & 0.4 \\
\hline Brachial tortuosity & $10(9 \%)$ & $139(9 \%)$ & 1 & $139(10 \%)$ & $10(5 \%)$ & 0.01 \\
\hline B group & $21(18 \%)$ & $362(24 \%)$ & 0.1 & $325(23 \%)$ & $58(27 \%)$ & 0.2 \\
\hline Axillary or subclavian tortuosity & $18(16 \%)$ & $282(19 \%)$ & 0.4 & $260(19 \%)$ & $40(19 \%)$ & 1 \\
\hline Axillary or subclavian stenosis or occlusion & $5(4 \%)$ & $91(6 \%)$ & 0.5 & $73(5 \%)$ & $23(11 \%)$ & 0.003 \\
\hline C group & $1(0.8 \%)$ & $44(3 \%)$ & 0.3 & $39(3 \%)$ & $6(3 \%)$ & 1 \\
\hline Lusoria & $1(0.8 \%)$ & $36(2 \%)$ & 0.5 & $34(2 \%)$ & $3(1 \%)$ & 0.4 \\
\hline Aortic elongation & 0 & $8(0.5 \%)$ & 1 & $5(0.3 \%)$ & $3(1 \%)$ & 0.07 \\
\hline
\end{tabular}

Table 3. Study endpoints in the two study groups and in PCI subgroups

\begin{tabular}{|c|c|c|c|c|c|c|}
\hline & $S G(n=112)$ & CG $(n=1,482)$ & $p$-value & PCI SG (n=82) & PCI CG $(n=257)$ & $p$-value \\
\hline Procedure success (\%) & $112(100 \%)$ & $1,274(86 \%)$ & 0.0001 & $82(100 \%)$ & $221(85.9 \%)$ & 0.001 \\
\hline Procedure duration (min) & $81 \pm 34$ & $60 \pm 32$ & 0.001 & $87 \pm 39$ & $87 \pm 37$ & 0.8 \\
\hline Fluoroscopic time (min) & $20 \pm 13$ & $13 \pm 9$ & 0.001 & $21 \pm 15$ & $21 \pm 11$ & 0.7 \\
\hline Total DAP & $55,985 \pm 70,320$ & $97,770 \pm 92,872$ & 0.001 & $60,686 \pm 74,907$ & $129,765 \pm 122,395$ & 0.001 \\
\hline Contrast media (ml) & $220 \pm 109$ & $160 \pm 101$ & 0.001 & $236 \pm 111$ & $265 \pm 105$ & 0.03 \\
\hline Vascular complication & $2(1.7 \%)$ & $47(3 \%)$ & 0.5 & $1(1 \%)$ & $9(3 \%)$ & 0.4 \\
\hline Need for a buddy wire & $4(3.5 \%)$ & $8(0.5 \%)$ & 0.4 & $4(4.8 \%)$ & $8(3.1 \%)$ & 0.4 \\
\hline Need for a guiding catheter extension & $3(2.6 \%)$ & $3(0.2 \%)$ & 0.1 & $3(3.6 \%)$ & $3(1.2 \%)$ & 0.1 \\
\hline \multicolumn{7}{|l|}{ DAP: dose area product } \\
\hline
\end{tabular}


Table 4. Study endpoints in the sheathless and 6 Fr and 5 Fr guiding catheter groups in PCI procedures.

\begin{tabular}{|c|c|c|c|c|c|}
\hline & $\begin{array}{l}\text { PCI SG } 6.5 \text { and } \\
7.5 \mathrm{Fr}(n=82)\end{array}$ & $\begin{array}{c}\text { PCI CG } 6 \text { Fr } \\
(n=247)\end{array}$ & $p$-value* & $\begin{array}{c}\text { PCI CG } 5 \text { Fr } \\
(n=12)\end{array}$ & $p$-value ${ }^{\pi}$ \\
\hline Procedure success (\%) & $82(100 \%)$ & $213(86.2 \%)$ & 0.001 & $8(66.6 \%)$ & 0.001 \\
\hline Procedure duration (min) & $87 \pm 39$ & $87 \pm 37$ & 0.9 & $75 \pm 35$ & 0.3 \\
\hline Fluoroscopic time (min) & $21 \pm 15$ & $21 \pm 11$ & 0.9 & $16.5 \pm 8.3$ & 0.3 \\
\hline Total DAP & $60,686 \pm 74,907$ & $132,975 \pm 122,893$ & 0.001 & $30,277 \pm 25,264$ & 0.3 \\
\hline Contrast media (ml) & $236 \pm 111$ & $268 \pm 105$ & 0.02 & $210 \pm 80$ & 0.4 \\
\hline Vascular complication & $1(1 \%)$ & $8(3.2 \%)$ & 1 & $1(8.3 \%)$ & 0.3 \\
\hline Need for a buddy wire & $4(4.8 \%)$ & $8(3.2 \%)$ & 0.5 & $0(0 \%)$ & 1 \\
\hline Need for a guiding catheter extension & $3(3.6 \%)$ & $3(1.2 \%)$ & 0.1 & $0(0 \%)$ & 1 \\
\hline
\end{tabular}

failure rate of TRA in coronary procedures is $5.8 \%{ }^{2}$ and it is significantly higher than that reported in the TFA. The TRA is associated with a number of difficulties such as catheter friction, attributable to the small diameter or occurrence of spasm, and anatomical variations which have to be navigated to reach the ascending aorta. Limitations such as small radial artery size or the occurrence of spasm may be resolved using hydrophilic catheters for TRA procedures. Indeed, Koga et al have demonstrated that the use of hydrophilic catheters reduces radial artery spasm upon insertion, manipulation, and withdrawal of the catheter, as compared with non-hydrophilic catheters. In the sheathless guiding catheter system, a sheathless GC, which does not require an introducer, and has a hydrophilic coating along the whole length, allows the TRA in patients with small radial arteries, reducing frictional forces, discomfort and pain-induced radial artery $\operatorname{spasm}^{16}$. Youn et al have demonstrated the feasibility of sheathless GC during PCI procedures in patients with small radial artery size (diameter $<2.3 \mathrm{~mm}$ ), with a procedural success rate comparable to that of transfemoral PCI, in addition to reduced bleeding and vascular complication rates ${ }^{17}$. Recently sheathless guiding catheters have also been proven to be a safe, effective method for complex PCI via TRA in small radial arteries without catheter-related complications ${ }^{18}$. The gain in inner diameter of the sheathless guiding catheter system, as well as the hydrophilic coating, allows the use of a small radial artery for a vascular approach in complex PCI and can also reduce the risk of radial artery occlusion. This complication occurs, on average, in $5-12 \%$ of patients undergoing TRA procedures, and several studies have demonstrated that a sheath-to-artery ratio $>1$ is an independent predictor of radial artery occlusion ${ }^{19}$. The sheathless catheter system allows a catheter downsize; indeed the external diameter of a $6.5 \mathrm{Fr}(2.16 \mathrm{~mm})$ sheathless $\mathrm{GC}$ is smaller than that of a conventional $5 \mathrm{Fr}$ GC and, similarly, the external diameter of a $7.5 \mathrm{Fr}(2.49 \mathrm{~mm})$ sheathless $\mathrm{GC}$ is smaller than that of a 6 Fr GC (2.7 mm) (Figure 1).

A previous study demonstrated that TRA failure was more frequently associated with upper arm vascular anomalies (15.6\%) than with radial artery spasm $(0.7 \%)$. In the presence of documented variants, the cause of TRA failure was unsuccessful advancement of catheters into the radial/brachial artery and ascending aorta ${ }^{20}$. Our data confirmed that in a high volume centre, procedural failure of TRA is associated with a $360^{\circ}$ radial loop and brachial tortuosity. This retrospective study has also demonstrated that the sheathless system may be used to resolve navigation difficulties in reaching the ascending aorta in the presence of upper arm vascular anomalies. Indeed, in our study, the use of a sheathless GC is associated with a higher incidence of procedural success as compared to use of a conventional catheter. Nevertheless the use of a sheathless GC is associated with a higher procedure duration, fluoroscopic time and amount of contrast medium in all (diagnostic and interventional) study procedures. The sheathless GC was used in diagnostic procedures where there was evidence of upper limb vascular anomalies and where the manipulation of the guiding catheter might be more complex as compared to conventional diagnostic catheters. Use of the sheathless GC is associated with a higher procedural success rate. In a sub-analysis of PCI procedures, use of a sheathless system is associated with a lower dose area product (DAP) and amount of contrast medium used. In PCI procedures, the procedural success advantage of sheathless GC is independent of conventional guiding catheter size. Therefore, the usefulness and safety of sheathless GC has been demonstrated in resolving the navigation difficulties in reaching the ascending aorta in the presence of upper arm vascular anomalies, not only for PCI procedures, but also for diagnostic procedures.

Previous experience has demonstrated that the sheathless GC allows effective cannulation of the ostium, but it is not as efficient as conventional catheters in terms of support. In our study the incidence of the use of additional techniques to complete the procedures, such as guiding catheter extension or buddy wire to increase the guiding catheter support, is not different between SG and CG. Probably the good selection of the most suitable catheter shape might have helped to obtain the best possible support.

The TRA is associated with a relevant reduction in vascular complications and this reduction may limit major clinical adverse events in some patients, for instance those with ST-elevation myocardial infarction ${ }^{2,21}$. In our study, the incidence of vascular complications was not different between the SG and CG. However, 
female gender with small radial artery and the incidence of unstable clinical presentation were more frequent in the SG as compared to the CG. The selection bias of a non-randomised study might have underestimated the beneficial effects of a sheathless $\mathrm{GC}$ on vascular complications.

\section{Limitations}

This is a non-randomised, observational study, the results of which may have been affected by a selection bias. In particular, in the study the decision to use a sheathless GC, instead of a conventional catheter was left to the interventional cardiologist's discretion after finding evidence of an upper limb vascular anomaly. However, although the use of this device was at the discretion of each operator, it became increasingly and almost automatically used in particular with upper arm anomalies such as a high origin radial artery. In two diagnostic procedures the sheathless GC was used after failure of a conventional catheter. The sheathless $\mathrm{GC}$ was intended to be systematically used in all diagnostic procedures and in all PCI procedures of the SG.

\section{Conclusions}

The TRA for coronary diagnostic and interventional procedures is known to shorten hospitalisation and dramatically reduce accesssite complications. On such a basis, TRA is now increasingly adopted in coronary and peripheral interventions. Nevertheless, TRA technical failure is significantly higher than that reported in the TFA. Such a high failure rate of TRA is due to a series of factors. In particular, a wide range of anatomic variants either of the brachioradial and the axillo-subclavian-anonymous arterial axis, or of the aortic arch may be present in patients undergoing TRA procedures, and these variants hinder procedural success. Our study has demonstrated that the SGC system is a safe and useful method not only in small radial access but also in the presence of upper vascular anomalies, and it can be used in PCI and diagnostic procedures.

\section{Impact on daily practice}

The TRA for diagnostic and interventional procedures reduces hospitalisation and access-site complications as compared to the TFA. Nevertheless, TRA failure is significantly higher than the TFA. Radial artery spasm and upper vascular anatomic variants influence the TRA technical procedural success. Device improvements are being developed to overcome these TRA limitations. An SGC, which does not require an introducer, has been developed in order to allow a gain in inner diameter and the hydrophilic coating reduces frictional forces, radial artery spasm and anatomic variant hinderance to navigation. Indeed the sheathless guiding catheter system is a safe and useful system not only for small radial access but also in the presence of upper vascular anomalies. The major advantages of the SGC system are in PCI procedures but can also be useful in diagnostic procedures in terms of procedural success.

\section{Conflict of interest statement}

F. Burzotta has been involved in advisory board meetings or has received speaker's fees from Abbott, Abiomed, Medtronic and Biotronic. C. Trani has been involved in advisory board meetings or has received speaker's fees from Abbott, Abiomed, Medtronic and Biotronic. C. Aurigemma has been involved in advisory board activities or has received speaker's fees from Abbott, Abiomed, Medtronic and Biotronic. A.M. Leone has received speaker's fees from Abbott, Abiomed and Bracco. I. Porto has been involved in advisory board activities or has received speaker's fees from Abbott, Abiomed and Biotronic. G. Russo reports grants from Edwards Lifesciences, outside the submitted work The other authors have no conflicts of interest to declare.

\section{References}

1. Agostoni P, Biondi-Zoccai GG, de Benedictis ML, Rigattieri S, Turri M, Anselmi M, Vassanelli C, Zardini P, Louvard Y, Hamon M. Radial versus femoral approach for percutaneous coronary diagnostic and interventional procedures; systematic overview and meta-analysis of randomized trials. J Am Coll Cardiol. 2004;44: 349-56.

2. Jolly SS, Amlani S, Hamon M, Yusuf S, Mehta SR. Radial versus femoral access for coronary angiography or intervention and the impact on major bleeding and ischemic events: a systematic review and meta-analysis of randomized trials. Am Heart J. 2009;157: 132-40.

3. Hildick-Smith DJ, Ludman PF, Lowe MD, Stephens NG, Harcombe AA, Walsh JT, Stone DL, Shapiro LM, Schofield PM, Petch MC. Comparison of radial versus brachial approaches for diagnostic coronary angiography when the femoral approach is contraindicated. Am J Cardiol. 1998;81:770-2.

4. Sciahbasi A, Romagnoli E, Trani C, Burzotta F, Pendenza G, Tommasino A, Leone AM, Niccoli G, Porto I, Penco M, Lioy E. Evaluation of the "learning Curve" for left and right radial approach during percutaneous coronary procedures. Am J Cardiol. 2011; 108:185-8.

5. Burzotta F, Trani C, Hamon M, Amoroso G, Kiemeneij F. Transradial approach for coronary angiography and interventions in patients with coronary bypass grafts: tips and tricks. Catheter Cardiovasc Interv. 2008;72:263-72.

6. Trani C, Tomassino A, Burzotta F. Transradial renal stenting: why and how. Catheter Cardiovasc Interv. 2009;74:951-6

7. Trani C, Burzotta F, Tomassino A, Giammarinaro M. Transradial approach to treat superficial artery in-stent restenosis. Catheter Cardiovasc Interv. 2009;74:494-8.

8. Trani C, Burzotta F, Coroleu GF. Transradial carotid artery stenting with proximal embolic protection. Catheter Cardiovasc Interv. 2009;74:267-72.

9. Mamas M, D’Souza S, Hendry C, Ali R, Iles-Smith H, Palmer K, El-Omar M, Fath-Ordoubadi F, Neyses L, Fraser DG. Use of the sheathless guide catheter during routine transradial percutaneous coronary intervention: a feasibility study. Catheter Cardiovasc Interv. 2010;75:596-602. 
10. Romagnoli E, Burzotta F, Trani C, Siviglia M, BiondiZoccai GG, Niccoli G, Leone AM, Porto I, Mazzari MA, Mongiardo R, Rebuzzi AG, Schiavoni G, Crea F. EuroSCORE as predictor of in-hospital mortality after percutaneous coronary intervention. Heart. 2009;95:43-8.

11. Saffioti S, Coluccia V, Burzotta F, Trani C, Niccoli G, Leone AM, Aurigemma C, Crea F. Value of EuroSCORE II in predicting total and cardiac mortality in patients undergoing percutaneous coronary interventions. Am J Cardiol. 2014;113:745-6.

12. Burzotta F, Trani C, Mazzari MA, Tommasino A, Niccoli G, Porto I, Leone AM, Tinelli G, Coluccia V, De Vita M, Brancati M, Mongiardo R, Schiavoni G, Crea F. Vascular complications and access crossover in 10,676 transradial percutaneous coronary procedures. Am Heart J. 2012;163:230-8.

13. Barbeau GR, Arsenault F, Dugas L, Simard S, Larivière MM. Evaluation of the ulnopalmar arterial arches with pulse oximetry and plethysmography: Comparison with the Allen's test in 1010 patients. Am Heart J. 2004;147:489-93.

14. Burzotta F, Trani C, De Vita M, Crea F. A new operative classification of both anatomic vascular variants and physiopathologic conditions affecting transradial cardiovascular procedures. Int $J$ Cardiol. 2010;145:120-2.

15. Burzotta F, De Vita M, Trani C. How to manage difficult anatomic conditions affecting transradial approach coronary procedures? Indian Heart J. 2010;62:238-244.

16. Koga S, Ikeda S, Futagawa K, Sonoda K, Yoshitake T, Miyahara Y, Kohno S. The use of a hydrophilic-coated catheter during transradial cardiac catheterization is associated with a low incidence of radial artery spasm. Int J Cardiol. 2004;96:255-8.

17. Youn YJ, Yoon J, Han SW, Lee JW, Sung JK, Ahn SG, Kim JY, Yoo BS, Lee SH, Choe KH. Feasibility of transradial coronary intervention using a sheathless guiding catheter in patients with small radial artery. Korean Circ J. 2011;41:143-8.

18. Cheaito R, Benamer H, Hovasse T, Tritar A, Hage F, Garot P, Lefèvre T, Unterseeh T, Chevalier B, Morice MC, Louvard Y. Feasibility and safety of transradial coronary interventions using a 6.5-F sheathless guiding catheter in patients with small radial arteries. Catheter Cardiovasc Interv. 2015;86:51-8.

19. Buturak A, Gorgulu S, Norgaz T, Voyvoda N, Sahingoz Y, Degirmencioglu A, Dagdelen S. The long-term incidence and predictors of radial artery occlusion following a transradial coronary procedure. Cardiol J. 2014;21:350-6.

20. Burzotta F, Brancati MF, Trani C, Tommasino A, Porto I, Niccoli G, Leone AM, Coluccia V, Di Noi P, Crea F. Impact of radial-to-aorta vascular anatomical variants on risk of failure in trans-radial coronary procedures. Catheter Cardiovasc Interv. 2012;80:298-303.

21. Jolly SS, Yusuf S, Cairns J, Xavier D, Widimsky P, Budaj A, Niemelä M, Valentin V, Lewis BS, Avezum A, Steg PG, Rao SV, Gao P, Afzal R, Joyner CD, Chrolavicius S, Mehta SR; RIVAL trial group. Radial versus femoral access for coronary angiography and intervention in patients with acute coronary syndromes (RIVAL): a randomised, parallel group, multicentre trial. Lancet. 2011;377: 1409-20. 